\title{
Surface characterization of imprinted resist above glass transition temperature
}

\author{
T. Lévéder and S. Landis ${ }^{\mathrm{a})}$ \\ CEA LETI-Minatec, 17 Rue des Martyrs, 38054 Grenoble, France \\ L. Davoust \\ LEGI/ENSHMG, 1025 Rue de la Piscine, Domaine Universetaire, BP 53, 38041 Grenoble, France
}

\author{
S. Soulan, J.-H. Tortai, and N. Chaix \\ CNRS-LTM, 17 Rue des Martyrs, 38054 Cedex 9, Grenoble, France
}

(Received 8 June 2007; accepted 24 September 2007; published 10 December 2007)

\begin{abstract}
Nanoimprint lithography is a high resolution and low cost patterning technique. Many difficulties have been overcome from the process point of view. This article is dedicated to the resist pattern characterization when they are annealed above the glass transition temperature of the material. This approach may be directly transposed to imprint process optimization when the demolding scheme is performed above the glass transition temperature. Simple model describing shape evolution with temperature is proposed and correlation with demolding process is presented. The results showed that a stamp release well above glass transition temperature is possible and may slightly impact the initial imprinted shape. Furthermore, this work may be transposed to determine temperature evolution of polymer dynamic viscosity. () 2007 American Vacuum Society. [DOI: 10.1116/1.2799975]
\end{abstract}

\section{INTRODUCTION}

Whereas wavelength downsizing or immersion techniques are proposed to improve capabilities of conventional optical projection lithography, other approaches are proposed for next generation lithography. Nanoimprint lithography (NIL) is emerging as the long-awaited disruptive technology. Among them, the hot embossing (HE) lithography, proposed by Chou et al. in $1995,{ }^{1}$ has now achieved a sufficient maturity level to be implemented in production scale. Molecular-scale resolution recently ${ }^{2}$ demonstrated that the main issues of NIL are not now more resolution but defectivity and throughput.

Even if complex three-dimensional (3D) features may be imprinted in one step ${ }^{3}$ one of the main industrial issues remains the throughput of HE processes with respect to current lithography approaches. In this article we focus onto the resist relaxation scheme when demolding processes may be performed above or below the glass transition temperature $\left(T_{g}\right)$ of the resist in order to reduce the cooling time of the whole imprint setup. We will show how the temperature cycle of imprint process-i.e., imprinting temperature, cooling down rate, and demolding temperature-may be optimized. Thus both imprinting and stamp release processes may be time optimized to increase the HE throughput.

Our experimental approach will be first detailed and then a complete atomic force microscopy (AFM) characterization of reflowing resist pattern will be analyzed. Both time and temperature annealing effect will be characterized. First results of a scatterometry approach linked to thermal and mechanical properties of material will be presented. We will

a) Author to whom correspondence should be addressed; electronic mail: slandis@cea.fr explain how this may reach a real time in-line reflow characterization technique. As a consequence, we will show that our experimental approach may be used not only for process optimization but also for fundamental characterization of melt polymer flow properties.

\section{EXPERIMENT}

The printing processes have been performed on EVG®520HE system ${ }^{4}$ for substrate sizes up to $200 \mathrm{~mm}$ wafers. The maximum applied force is $40 \mathrm{kN}$, equivalent to a pressure of 13 bars on a whole $200 \mathrm{~mm}$ wafer. The printing process was performed under vacuum by pumping the chamber down to $5 \times 10^{-4}$ bar.

In order to characterize and analyze the potential surface reflow during demolding, our approach consists in the following: first, imprinting the resist with a stamp release well below $T_{g}$ to prevent from any reflow; then performing successive annealing, quenching at room temperature, and characterizations of the resist relaxations (Fig. 1). Frozen shapes of patterns were measured with an atomic force microscopy setup. A $200 \mathrm{~mm}$ silicon stamp with $250 \mathrm{~nm}$ dense line array, $180 \mathrm{~nm}$ deep, has been printed into $100 \mathrm{~nm}$ thick mr-I 7010E resist from Micro Resist Technology. By volume conservation of the imprinted polymer, it can be determined how thick has to be the thinnest film that can be imprinted. Indeed, a two-dimensional (2D) isodense (50\%) $180 \mathrm{~nm}$ deep mold required $(180 \times 50) \%=90 \mathrm{~nm}$ of resist. With a $100 \mathrm{~nm}$ thick resist film, we ensure full filling of the patterns. Printings were performed at $110{ }^{\circ} \mathrm{C}\left(T_{g}+50{ }^{\circ} \mathrm{C}\right)$ under $40 \mathrm{kN}$ for 2 min.

Accurate measurements of the reflowing shape are tricky, even if the measurement is performed onto the frozen states. Indeed, for slight resist reflowing (short annealing times) the 


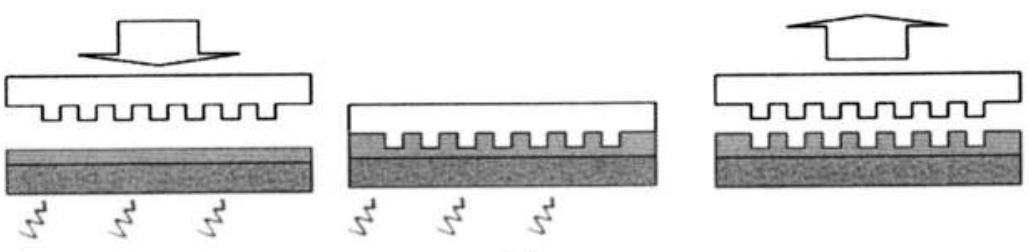

(a)
FIG. 1. (a) Imprint at temperature $(T)$ above $T_{g}$ and demolding scheme ( $T$ $<T_{g}$ ). (b) Postdemolding backing step and lines reflowing at temperature above $T_{g}$.

(above

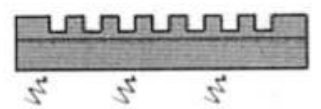

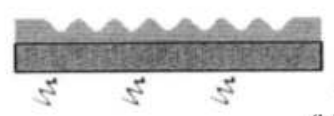

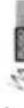

(b)

b)
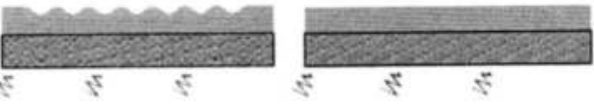

pattern sidewalls were larger than $70^{\circ}$. Flared tips onto Veeco AFM X3D were therefore used as long as the side angle was larger than $77^{\circ}$. Such tips can access reentrant patterns but not acute angles. ${ }^{5}$ Then, for smoother profiles standard conic tips are sharp enough to explore the bottom of the trenches.

The first stages of the reflow are thus characterized with flared tips [Fig. 2(a)] whereas the last were measured with conic tips [Fig. 2(b)]. Consequently, we could access accurate measurements of the whole evolution of a reflowing grating as a function of time. Those measurements have been performed for four different temperatures: $80,90,100$, and $110{ }^{\circ} \mathrm{C}$. That is to say from 20 to $50^{\circ} \mathrm{C}$ above the glass transition temperature of the $\mathrm{mr}-\mathrm{I} 7000 \mathrm{E}$ resist.

\section{RESULTS AND ANALYSIS}

Figure 3(a) shows the cross-sectional profile evolution for a $180 \mathrm{~nm}$ deep, $250 \mathrm{~nm}$ dense line array annealed at $80^{\circ} \mathrm{C}$ $\left(T_{g}+20^{\circ} \mathrm{C}\right)$ from 1 to $500 \mathrm{~s}$. Note that conic tips were used to explore the bottom of the trenches for annealing time larger than $100 \mathrm{~s}^{6}$ Increasing the annealing time, the crosssectional profiles become more rounded and pattern height decreased. The surface free energy is clearly the driving force of such phenomenon as it tends to minimize the interfacial surface between resist and air.

Similar results are obtained when the annealing temperature is increasing as shown in Fig. 3(b). The cross-sectional profiles became more and more rounded when the annealing temperature gets higher. In such reflow behavior, polymer dynamic viscosity is known to play a key role since it slows down the material motion. Therefore the balance between surface free energy and dynamic viscosity defines the velocity of the reflow process.

$$
\frac{\sigma}{\eta} \equiv \frac{\left[\mathrm{J} \mathrm{m}^{-2}\right]}{[\mathrm{Pa} \mathrm{s}]}=\frac{\left[\mathrm{N} \mathrm{m}^{-1}\right]}{\left[\mathrm{N} \mathrm{s} \mathrm{m}^{-2}\right]}=\mathrm{m} \mathrm{s}^{-1} .
$$

Since the polymer surface free energy is known not to be a parameter very sensitive to temperature range we explored, our results indicate that the dynamic viscosity, as expected, is temperature dependant. Consequently, these experiments may be used both to determine process windows in order to perform demolding at higher temperature than below $T_{g}$ and to determine the temperature-polymer viscosity relationship, assuming the surface free energy is temperature independent. ${ }^{8}$

Given thermal inertia of silicon samples, the annealing temperature for our study is reached before $4 \mathrm{~s}$, which means that the error made onto the real annealing is well below $10 \%$ for the shortest time and below $0.5 \%$ for the longer ones. Moreover as the viscosity is very sensitive to temperature offset with respect to $T_{g}$, we can assume that the kinetic of shape modification is much slower during the temperature raise than the kinetic at the final annealing temperature. Therefore, the potential shape difference between a reannealed sample (i.e., our experiment) and a demolded sample will be in the uncertainty of the characterization technique used and does not affect our results.

Figure 4 shows the evolution of the pattern height, i.e., the difference between the highest and lowest points in the cross-sectional profiles, determined for annealing temperature between 80 and $110{ }^{\circ} \mathrm{C}$ and annealing time up to $500 \mathrm{~s}$ (Fig. 4). Each experimental point corresponds to an average value over 20 scan lines onto three printed resist lines with a deviation from the mean value lower than $2 \%$. Figure 4 clearly demonstrates that annealing temperature has a much larger effect onto the resist reflow than the annealing time.

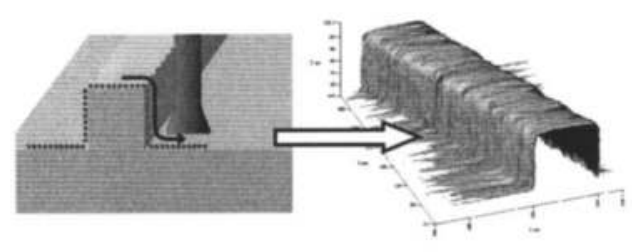

(a)

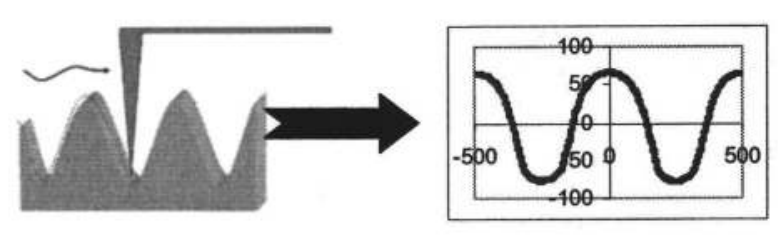

(b)

FIG. 2. AFM measurements (a) with flare tips for steep resist profiles and (b) with conic tips for smoother resist profiles. 

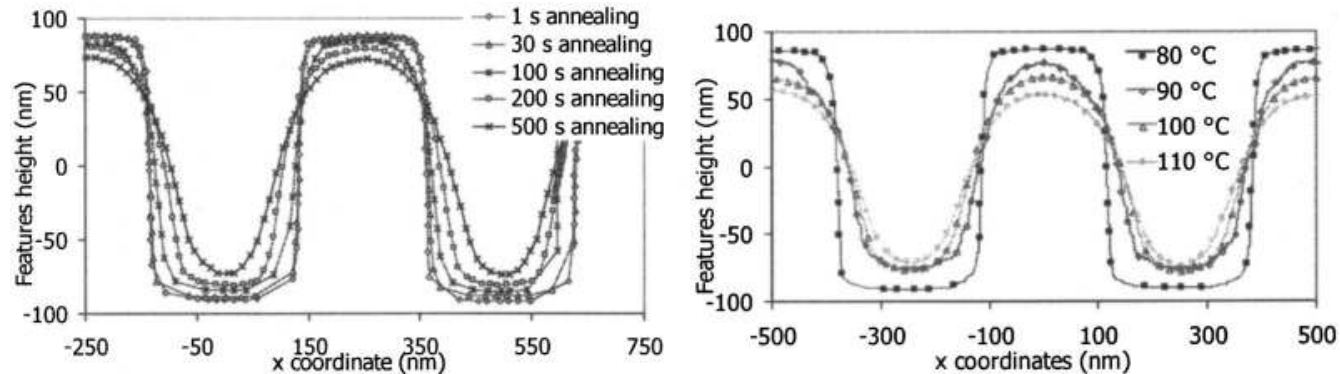

FIG. 3. AFM cross-sectional profiles of annealed resist patterns. The annealing temperature and time were $80{ }^{\circ} \mathrm{C}$ and up to $500 \mathrm{~s}$, respectively (left curves). For the right figure the annealing time has been fixed at $30 \mathrm{~s}$ and the temperature was ranging from $80{ }^{\circ} \mathrm{C}$ up to $110^{\circ} \mathrm{C}$.

The linear-log scale also reveals that the experimental evolution of the resist feature height may be well fitted with an exponential law such as $h(t)=h_{0} e^{-(t / \tau)}$, where $h_{0}$ is the initial resist height of the pattern and $\tau$ a characteristic time of the resist reflow. Insets in Fig. 4 also show that other geometrical parameters of the resist patterns (sidewall angle or top rounding) are well fitted with exponential law (Fig. 4). This suggests that the whole pattern shape evolution, when the temperature is constant, may be defined by a quality factor $Q(t, T)=\exp (-t / \tau(T))$. In real configuration the temperature cannot be considered as constant during a quenching so that $Q(t, T)$ could not be directly plotted to represent the shape evolution. Nevertheless, this complex time-thermal path may be approximated by successive isothermal steps.

$$
Q=\int d Q \approx \sum_{i} \int_{t_{i}}^{t_{i+1}} \frac{\partial Q(t, T)}{\partial t} d t+\int_{T_{i}}^{T_{i+1}} \frac{\partial Q(t, T)}{\partial T} d T .
$$

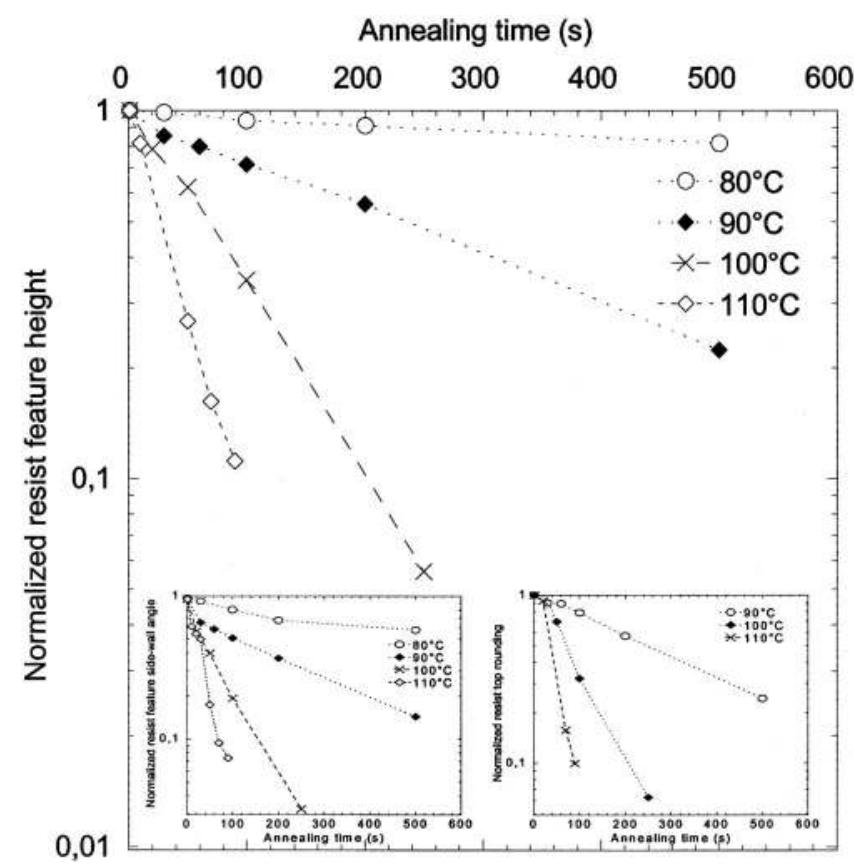

FIG. 4. Feature height variation with respect to annealing time. Annealings at $80,90,110$, and $110{ }^{\circ} \mathrm{C}$ were performed onto the printed lines. The insets represent also the evolution of sidewall angle and top corner rounding of the lines, respectively.
Assuming $T_{i}+1 \sim T_{i}$ for short time step, the quality factor could be numerically computed with a given temperaturetime dependency. Knowing the cooling rate that could be applied after demolding the stamp, it is therefore possible to predict the shape modification of the resist patterns. Figure 5 shows the quality factor evolution for a demolding at $120^{\circ} \mathrm{C}$ $\left(T_{g}+70{ }^{\circ} \mathrm{C}\right)$ for a cooling rate ranging from 1 to $3{ }^{\circ} \mathrm{C} / \mathrm{min}$, which implies a shape modification ranging from $2 \%$ to $7 \%$. This approach can be then applied to determine the process window for the demolding step: the temperature when the stamp should be release from the imprinted resist with respect to the imprinted temperature, glass transition temperature, and the system cooling rate, respectively.

The following section focuses onto the polymer flow property characterization. Following the approach proposed by Hamdorf and Johannsmann, ${ }^{9}$ the viscosity of the film may be estimated from the relation $\eta(T) \approx \pi \sigma \tau(T) /(2 \lambda)$, where $\lambda$ is the pattern wavelength and $\tau(T)$ the pattern relaxation time previously measured. Figure 4(b) shows the experimental evolution of the relaxation time $\tau$ of the pattern. The previous relationship between viscosity and relaxation time sug-

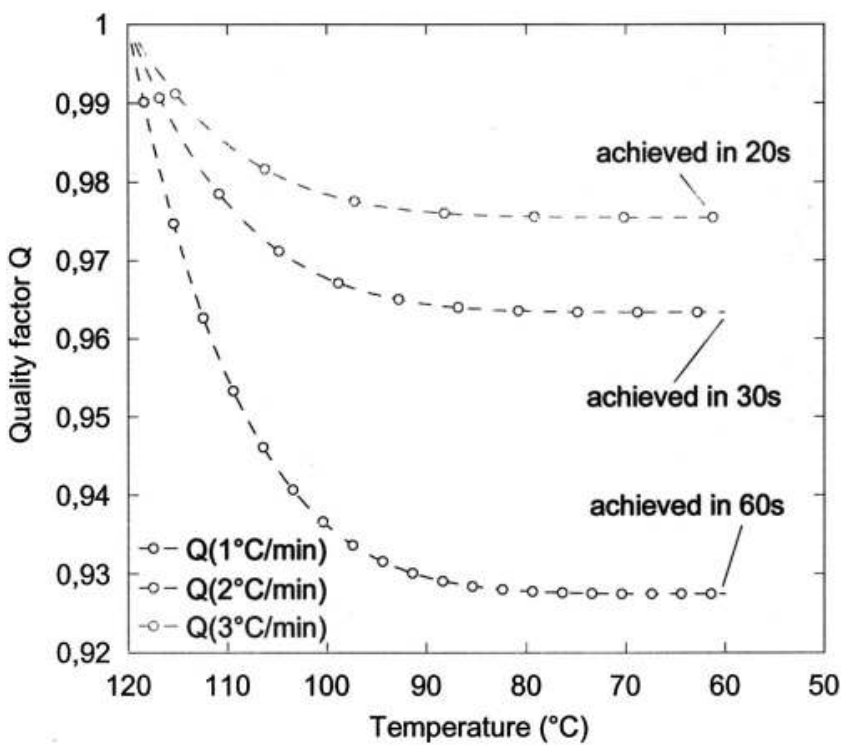

FIG. 5. Evolution of the quality factor $Q$ for quenching with rates of 1,2 , and $3{ }^{\circ} \mathrm{C} / \mathrm{min}$, respectively. These three curves are representative of the shape evolution during sample quenching when stamp is removed above $T_{g}$. 


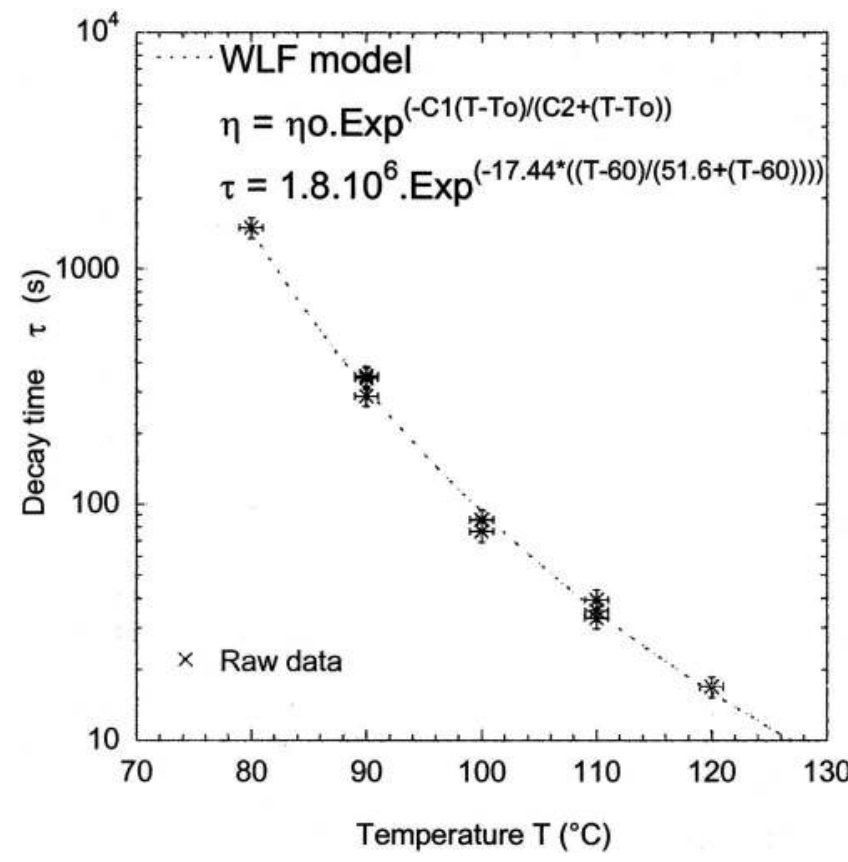

FIG. 6. Evolution of the relaxation time $\tau$ with respect to the temperature. Experimental points (crosses) were fitted using the WLF model (dashed curve) with $C_{1}=17.44, C_{2}=51.6$, and $T_{0}=T_{g}$, respectively.

gests that the Williams-Landel-Ferry (WLF) equation ${ }^{10}$ usually used to describe the temperature dependency of viscosity may be also applied to $\tau$.

$$
\begin{aligned}
\eta & =\eta_{0} \exp ^{\left(C_{1}\left(T-T_{0}\right) / C_{2}+\left(T-T_{0}\right)\right)} \Rightarrow \tau \\
& =\tau_{0} \exp ^{\left(C_{1}\left(T-T_{0}\right) / C_{2}+\left(T-T_{0}\right)\right)} .
\end{aligned}
$$

Using $C_{1}=17.44, C_{2}=51.3 \mathrm{~K}$, and $T_{0}=T_{g}=60{ }^{\circ} \mathrm{C},{ }^{10}$ Fig. 6 shows favorable agreement between the WLF model and the experimental evolution of relaxation time with temperature. Therefore, $\eta_{0}=\pi \sigma \tau_{0} /(2 \lambda)$ is equal to $2.8 \times 10^{11} \mathrm{~Pa} \mathrm{~s}$, which is close to the value found for similar polymer chemistry.

The study of pattern reflow is clearly a way to address both technological (demolding process) and fundamental (flow property characterization) issues. Nevertheless, it requires a perfect knowing of the shape evolution with respect to the temperature-time parameters. Our approach using atomic force microscopy is therefore time consuming. Another measurement principle based on scatterometry technique may be thus carried out to perform a real time characterization. Scatterometry technique is based on the analysis of the diffraction of light from periodic patterns and can address both 2D and 3D patterns. This is not an optical imaging technique but measurement and analysis of the diffracted coherent illumination of a sample. The inverse problem consists of determining the shape of the lines of the periodic structure which diffracts the incident light. The optimization step consists either of using a library-based methodology ${ }^{11}$ or of using standard optimization routines such as the Levenberg-Marquardt algorithm ${ }^{12,13}$ for structure size extraction. The best match between the experimental

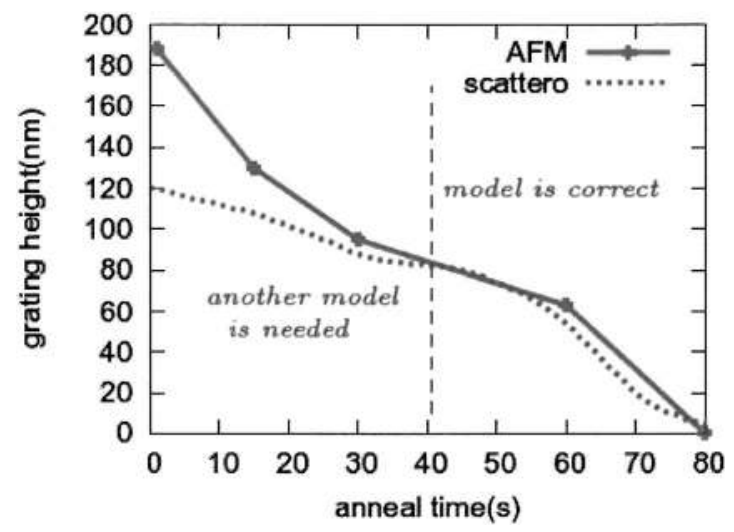

FIG. 7. Resist height evolution with respect to the annealing time at $T_{g}$ $+30{ }^{\circ} \mathrm{C}$. AFM measurements and scatterometry characterizations with sinusoidal shape model were both performed.

scatter signature and theoretically generated ones is sought by minimizing the absolute mean square error between these signatures. In this study we used the method of library searching, most commonly implemented in the industry. Moreover, this method is real time compliant as the solving time is perfectly predictable.

We looked to the pattern shape variation $(250 \mathrm{~nm}$ dense lines) during reflowing at $T_{g}+30{ }^{\circ} \mathrm{C}$ with an acquisition frequency of about 1 signature/s. For the library, we choose to model the pattern shape using a mere sine wave. Its amplitude is thus the only variable parameter. Figure 7 shows a comparison between reconstructed profile shape and AFM measurements made in the same conditions and at different time steps. We can clearly see that there are two regimes in Fig. 7. Below $40 \mathrm{~s}$ the AFM and scatterometry results do not match whereas for larger annealing time, the two techniques gave very close results. The main reason why the two curves do not match in the first stage is that the real grating shape is rectangular before the reflow, whereas a sine model was used fore the scatterometry library. A more accurate geometrical model would improve the matching between both techniques for the whole reflow process. Nevertheless, this preliminary study shows that the standard scatterometry is efficient and dynamic shape reconstruction can work.

\section{CONCLUSION}

We show in this article that a full characterization of resist reflow leads to an analysis which make predictive the imprinted resist behavior when the stamp release is performed above the glass transition temperature. As a consequence, the imprinted temperature should not be only optimized to reduce as much as possible the polymer viscosity (so the imprinting time). We demonstrate that knowing the resist reflow properties, the pattern reflow is predictable and that demolding above $T_{g}$ is therefore possible. The whole cycle time may be thus reduced, thanks to an optimization of both the imprint and release temperature with respect to the cooling rate of the imprint setup. To reduce the resist characterization time, we also show that scatterometry approach may save a 
large amount of time with respect to full 3D AFM measurements. Thanks to these fine morphological characterization resist flow properties, as dynamic viscosity may be also characterized and extrapolated from WLF theory.

${ }^{1}$ S. Y. Chou, P. R. Krauss, and P. J. Renstrom, Appl. Phys. Lett. 67, 3114 (1995).

${ }^{2}$ F. Hua et al., Nano Lett. 12, 2467 (2004).

${ }^{3}$ M. Tormen, F. Romanato, M. Altissimo, L. Businaro, P. Candeloro, and E. M. Di Fabrizic, J. Vac. Sci. Technol. B 22, 767 (2004).

${ }^{4}$ C. Perret, C. Gourgon, F. Lazzarino, J. Tallal, S. Landis, and R. Pelzer, Microelectron. Eng. 73-74, 172 (2004).

${ }^{5}$ G. Dahlen et al., J. Vac. Sci. Technol. B 23, 2297 (2005).
${ }^{6}$ Thanks to alignment capabilities of the AFM setup, measurements with both flared and conic tips were performed exactly and the same location. ${ }^{7}$ P.-G. de Gennes, F. Brochard-Wyart, and D. Quere, in Gouttes, Bulles, Perles et Ondes (Belin, 2005), pp. 127-137.

${ }^{8}$ I. Vilfan, F. Lançon, and E. Adam, Science 440, 279 (1999).

${ }^{9}$ M. Hamdorf and D. Johannsmann, J. Chem. Phys. 114, 9685 (2001); 112, 4262 (2000).

${ }^{10}$ J. D. Ferry, Viscoelastic Properties of Polymers, 3rd ed. (Wiley, New York, 1980).

${ }^{11}$ S. A. Coulombe, B. K. Minhas, and C. J. Raymond, J. Vac. Sci. Technol. B 16, 80 (1998).

${ }^{12}$ K. Levenberg, Q. Appl. Math. 2, 164 (1944).

${ }^{13}$ D. Marquardt, J. Soc. Ind. Appl. Math. 11, 431 (1963). 